\title{
Intrusion Detection Technique for Security Statistics
}

\author{
Sienna Arscott
}

\begin{abstract}
An Intrusion Detection System (IDS) is an hardware device or programming application that screens organize and additionally framework or host exercises for malevolent exercises or strategy infringement, makes and sends reports to a Management Station or System Administrator which concludes whether to make a move on the interruption or it was just a bogus alert. There are two kinds of Intrusion Detection Systems: Host based and Network based. System Intrusion Detection System (NIDS) distinguishes interruptions by inspecting system traffic and screens different hosts associated with the network.It catches all system traffic and investigates the substance of individual bundles for malicious traffic.
\end{abstract}

Keywords :IDS, cloud computing, service provider etc.

\section{INTRODUCTION}

The expression "cloud" in cloud computing is the communication arrange or a system joined with registering framework. It is an Internet-based figuring development, where shared resources, for instance, programming, stage, stockpiling and information are given to customers on demand. Cloud computing is an enlisting stage for sharing resources that join establishments, programming, applications, and business structures. Distributed computing is a virtual pool of preparing resources. It gives processing resources in the pool for customers through web.

Cloud computing is a perspective of coursed figuring to give the customers on-demand, utility based enlisting services. Cloud customers can give progressively reliable, open and revived services to their clients in this manner. Cloud itself includes physical machines in the server homesteads of cloud providers. Virtualization is given over these physical machines. These virtual machines are given to the cloud customers. Particular cloud provider gives cloud services of different consideration level. For instance Amazon EC2 engages the customers to manage low level nuances where Google App-Engine gives an improvement stage to the architects to develop their applications. So the cloud administrations are isolated into various sorts like Software as a Service, Platform as a Service or Infrastructure as a Service. These administrations are available over the Internet in the whole reality where the cloud goes about as the single reason for access for serving all customers. It configuration tends to difficulties of tremendous degree data getting ready. 


\section{Cloud Architecture}

The cloud providers truly have the physical server ranches to give virtualized services to their customers through Internet. The cloud providers consistently give division among application and data. This circumstance is showed up in the Figure. The basic physical machines are ordinarily made in grids and they are commonly topographically dispersed. Virtualization accept a critical activity in the cloud circumstance. The server ranch has give the physical hardware on which virtual machines abides. Customer possibly can use any OS maintained by the virtual machines used.



Fig: 1 Architecture of Cloud Computing

Operating system are expected for express equipment and programming. It achieves the absence of portability of working structure and programming beginning with one machine then onto the following machine which uses particular direction set building. The possibility of virtual machine deals with this issue by going about as an interface between the hardware and the working system called as structure VMs.Another arrangement of virtual machine is called process virtual machine which goes about as a one of a kind layer between the working structure and applications. Virtualization can be for the most part said to be as programming deciphering the hardware headings made by common programming to the reasonable association for the physical gear. Virtualization in like manner fuses the mapping of virtual resources like registers and memory to real gear resources. The concealed stage in virtualization is overall implied as host and the item that runs in the VM condition is called as the guest. The Figure shows very basics of virtualization. Here the virtualization layer covers the physical gear. Working System finds a good pace through virtualization layer. Applications can give direction by using OS interface similarly as clearly using virtualizing layer interface. This planempower the customers to use applications not impeccable with the OS.

Virtualization empowers the development of the virtual picture beginning with one physical machine then onto the following and this part is important for cloud as by data district heaps 
of progression is possible and besides this component is valuable for recovering up in different territories. This component furthermore enables the provider to close down a part of the server ranch physical machines to diminish power usage. The plan of Cloud incorporates different cloud parts talking with each other over the application programming interfaces (APIs), generally web administrations. The two most colossal pieces of distributed computing designing are known as the front end and the back end. The front end is the part seen by the client, for instance the customer. This joins the client's framework or PC, and the applications used to find a good pace through a UI, for instance, a web program. The back finish of the distributed computing configuration is essentially the 'cloud, which includes various PCs, servers and data storage devices.

\section{PROBLEMS WITH CURRENT CLOUD COMPUTING}

Coming up next are the major issues of current cloud processing structure:

I). Each service provider has its own product layer, stage layer and foundation layer. Right when a customer uses a cloud application from a service provider, the customer is constrained to use the stage and establishment gave by a comparable service provider, and thusly the service provider knows where the customers' data is found and has full access advantages to the data.

ii). The customer is constrained to use the interfaces just gave by the service provider, and customers' data must be in a fixed arrangement shown by the service provider, and along these lines the service provider understands all the information required understanding customers' data. Consequently, we can't thwart authority centers from satisfying the total of the three Conditions.

\section{Approach to Protect Confidentiality:}

In our methodology, we have the accompanying seven substances: Software Cloud, Infrastructure Cloud, Software Service Broker, Infrastructure Service Broker, Software Service Attestation Authority, Data Obfuscator and Data De-obfuscator 


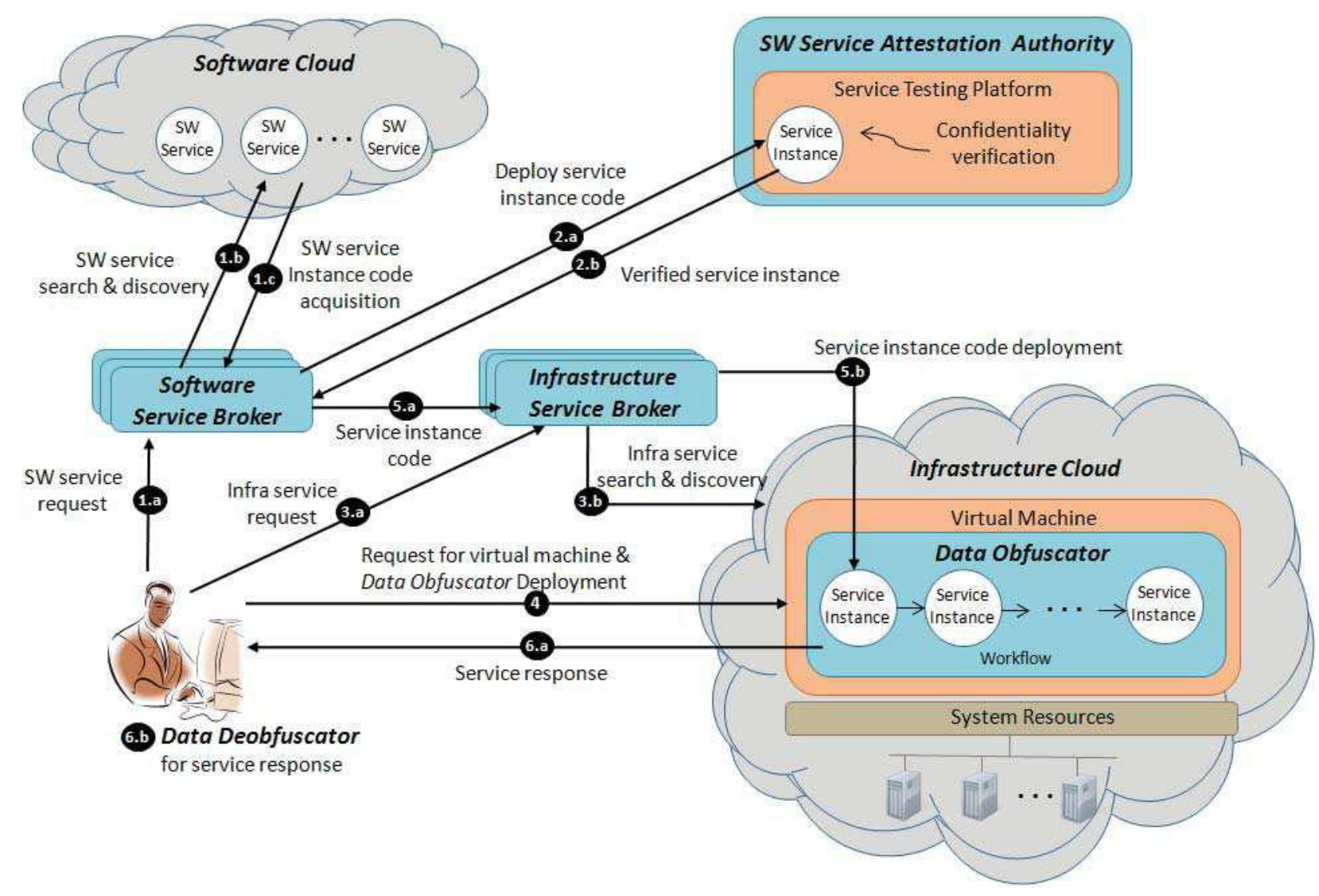

Fig: 2. Security in Cloud Computing

Our methodology guarantees that any of these substances in an appropriated processing system doesn't satisfy the three conditions simultaneously.

Software Cloud: A Software Cloud gives programming as a help upon customers' sales. Each item cloud may contain different programming administrations, and each item administration can be found and found a workable pace through Software Service Broker.

Software Cloud: An Infrastructure Cloud gives virtualized structure assets, for instance, CPU, memory, and framework resorces. A confirmed customer can request a virtual machine on which the customer can send any stage or working system to execute an item administration case.

Software Service Broker:It gives character anonymization administration, by which customers can use pseudonyms of their real characters with the objective that the customers can get administration events without revealing their characters. helps customers thusly find and use available establishment srvices. It moreover gives character anonymization administration to shield the structure from revealing customers' genuine characters.

The Software Service Attestation Authority (SSAA): The SSAA is an outsider position to check that an assistance occasion doesn't play out any pernicious movement that may uncover clients' private information 
A Data Obfuscator: A Data Obfuscator is a middleware given by a customer that can be sent on a virtual machine in an establishment Cloud. The Data Obfuscator gives a working system condition to programming service event to be run in an Infrastructure Cloud.

A Data De-obfuscator: It de-disorders tangled data with the objective that a customer can see the plain data. A Data Deobfuscator remains in the customer's PC continually.

\section{An Illustrative Example}

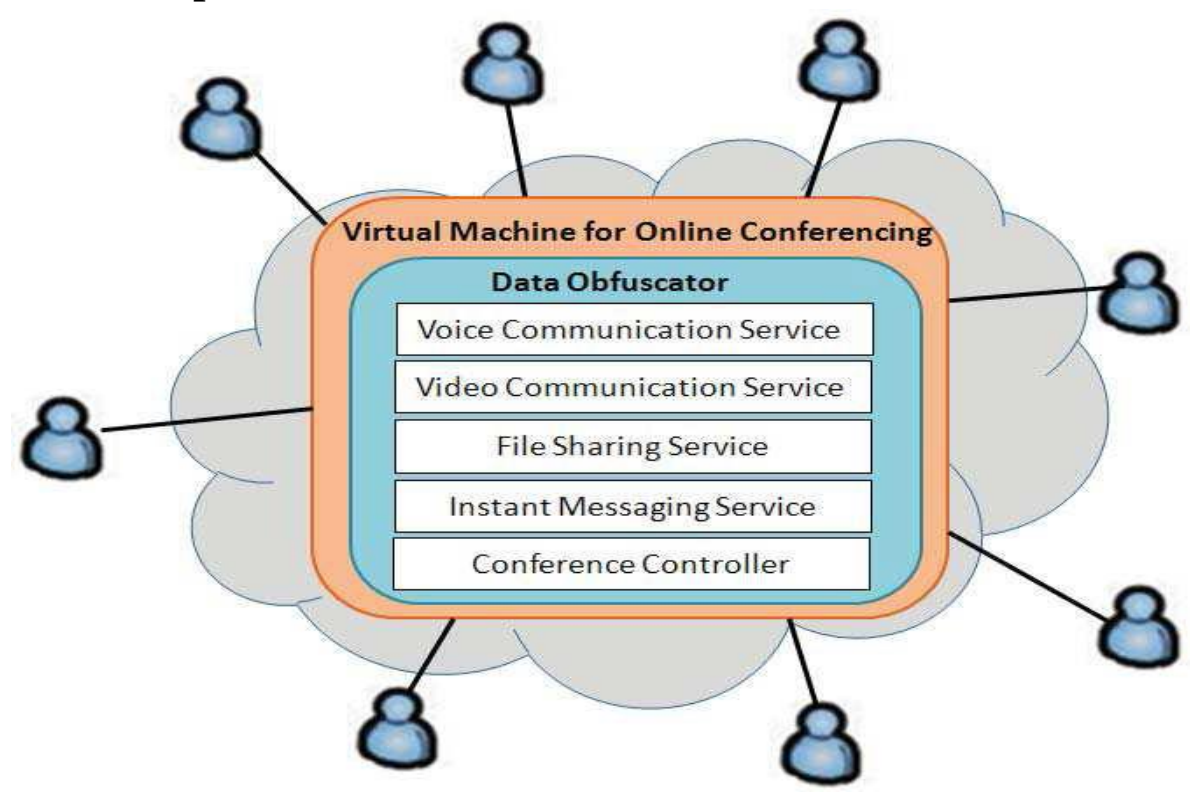

Fig 3 Basic Example

I) a) The pioneer of the social affair requests a Software Service Broker to find the Voice Communication Service, Video Communication Service, File Sharing Service and Instant Messaging Service.

b) The Software Service Broker finds the services.

c) The Software Service Broker downloads the services events of the five programming services an)

ii)a)The Software Service Broker sends the services events to the testing establishment of a SSAA.

b) The SSAA affirms the item services events.

iii) a) The pioneer of the get-together requests an Infrastructure Service Broker to find a structure services great to the administration events.

b) The Infrastructure Service Broker finds an establishment services.

iv) A virtual machine is set up in the establishment cloud. The pioneer of the get-together passes on the Data Obfuscator on the virtual Machine. 
v) a) The administration models are sent to the Infrastructure Service Broker.

b) The administration models are passed on the DataObfuscator. he five assistance events are framed to a work procedure. The work procedure gives all the functionalities to web conferencing.

vi) a) The customers of the get-together send their data to the work procedure to process. During the getting ready of the customers' data, the data is tangled. In the wake of completing the setting up, an assistance response of the work procedure is sent to all the customers of the social event that the treatment of their data has been done.

\section{DETECTION TECHNIQUE FOR THE IDS}

The proposed model of IDS targets recognizing numerous dangers which might happen in the cloud.

To do this it must have numerous standards by which it could characterize intrusion. Following rules for detecting an intrusion:

1. The patterns coordinate a current mark in the mark database.

2. Numerous mistaken passwords comparing to a solitary record or the framework in general could be an endeavor to break-in and can be named an interruption.

\section{Access right infringement:}

an) A client who is much of the time attempting to keep in touch with a document for which he has just understood access.

b) A client who is every now and again attempting to get to a document which he isn't approved to get to.

The above principles (an and b) speak to an infringement of access rights and can be forestalled by authorizing the Simple Security Condition of the Bell-LaPadulaModel and the Strict Integrity approach of the Biba Model.

4. Numerous cases of a solitary client (over a specific limit) getting to the framework simultaneously can be an aftereffect of effective break-in and masquerading attack.

5. Trojan Horse: The conduct of a Trojan steed planted in or fill in for a program may vary from the authentic program as far as CPU or I/O movement.

6. A refusal of service assault from a solitary machine or a cloud forswearing of service assault utilizing numerous 'synchronize' parcels can be recognized by examining bundles at the system layer and can be delegated an interruption. 
7. Spillage of information by authentic clients to other people who are not approved to see the information is an abuse of benefits and can be forestalled by applying the property of the Bell-LaPadula Model, and the Integrity Star property of the Biba Integrity model.

8. An authentic client login with exceptionally variation utilization than typical use may be an interruption. This standard has a high pace of bogus positives and consequently should be utilized related to different principles.

9. Topographical (spatial) and fleeting data about a client can likewise help in distinguishing interruptions.

\section{Data Confidentiality Protection}

Privacy is characterized as the affirmation that delicate data isn't revealed to unapproved people, procedures, or Devices.Users' classified information is uncovered to a specialist co-op if the entirety of the accompanying three conditions are fulfilled at the same time

1) The service provider knows where the clients' classified information is situated in the distributed computing frameworks.

2) The service provider has benefit to access and gather the clients' private information in cloud.

3) The service provider can comprehend the importance of the clients' information.

\section{CONCLUSION}

- A customer requests a Software Service Broker to find an item services by giving the assurance of the product services.

- $\quad$ The Software Service Broker performs modified help disclosure to find a help model in the Software Cloud that satisfies the customer's referenced assistance essential assurance.

- The Software Service Broker picks up the discovered programming event using an obscure capability.

- The Software Service Broker sends the picked up services guide to the testing establishment of a SSAA. The SSAA checks whether the services model proceeds as demonstrated by the services depiction, and the services case doesn't transmit customers' data to any unapproved component.

- After the check technique, the item services is sent back to the Software Service Broker.

- The customer asks the Software Service Broker to find an establishment services great to the service model. 
- The Infrastructure Service Broker finds an establishment authority association, who has the ability to execute the got programming's service model.

- The customer requests the establishment authority association to set up a virtual machine and a while later passes on the Data Obfuscator on the virtual machine using the Agent Deployment Plans (ADPs), for robotized middleware course of action and development in Software based systems

- $\quad$ The services event is sent on the work procedure of the Data Obfuscator set up in S4). The customer sends his/her data to the work procedure to process. During the getting ready of customers' data, the customer's data is cluttered with the objective that the structure authority association can't appreciate the significance of customer's data. Consequent to completing the setting up, an assistance response of the work procedure is sent to the customer indicating that the treatment of the customer's data has been done.

- $\quad$ The services response is de-jumbled to plain data in the customer's PC.

\section{REFERENCES}

[1] Mrs. Parag K. Shelke, Ms. SnehaSontakke, Dr. A.D.Gawande,'Intrusion Detection System for Cloud Computing", International Journal of Scientific \& Technology Research Volume 1, Issue 4, May 2012.

[2] Mrs. Parag K. Shelke, Ms. SnehaSontakke, Dr. A.D.Gawande,'Intrusion Detection System for Cloud Computing", International Journal of Scientific \& Technology Research Volume 1, Issue 4, May 2012.

[3] S. N. Pawar Associate Professor (E \&TC),Jawaharlal Nehru Engineering College, Aurangabad, MS, India. "INTRUSION DETECTION IN COMPUTER NETWORK USING GENETIC ALGORITHM APPROACH”, International Journal of Advances in Engineering \& Technology, May 2013.

[4] C. Modi, D. Patel, B. Borisaniya, H. Patel, A. Patel, M. Rajarajan, "A Survey of Intrusion Detection Techniques in Cloud", Journal of Network and Computer Applications 36 (2013), pp. 42-57.

[5] Monjurahmed and mohammadashrafhossain, senior lecturer,"Cloud Computing And Security Issues In The Cloud" daffodil institute of IT, Dhaka, Bangladesh, Vol.6, No.1, January 2014. 